\title{
Subadditive mean ergodic theorems
}

\author{
Y. DERRIENNIC AND U. KRENGEL $\dagger$ \\ Département de Mathématiques, Université de Bretagne Occidentale, Brest, France; \\ and Institüt für Mathematische Statistik, Göttingen, West Germany
}

(Received 24 November 1980)

\begin{abstract}
The authors investigate which results of the classical mean ergodic theory for bounded linear operators in Banach spaces have analogues for subadditive sequences $\left(F_{n}\right)$ in a Banach lattice $B$. A sequence $\left(F_{n}\right)$ is subadditive for a positive contraction $T$ in $B$ if $F_{n+k} \leq F_{n}+T^{n} F_{k}(n, k \geq 1)$. For example, von Neumann's mean ergodic theorem fails to extend to the general subadditive case, but it extends to the non-negative subadditive case. It is shown that the existence of a weak cluster point $f=T f$ for $\left(n^{-1} F_{n}\right)$ implies $\left\|\left(n^{-1} F_{n}-f\right)^{+}\right\| \rightarrow 0$. In $L_{p}(1 \leq p<\infty)$ the existence of a weak cluster point for non-negative $\left(n^{-1} F_{n}\right)$ is equivalent with norm convergence. If $T$ is an isometry in $L_{p}(1<p<\infty)$ and $\sup \left\{n^{-1}\left\|F_{n}\right\|_{p}\right\}<\infty$ then $n^{-1} F_{n}$ converges weakly. If $T$ in $L_{1}$ has a strictly positive fixed point and $\sup \left\{n^{-1}\left\|F_{n}\right\|_{1}\right\}<\infty$ then $n^{-1} F_{n}$ converges strongly. Most results are proved even in the $d$-parameter case.
\end{abstract}

\section{Introduction}

In this article we attempt to analyse which results of the classical mean ergodic theory for bounded linear operators in Banach spaces have analogues for subadditive sequences $F_{n}$ in Banach lattices $B$. A sequence $F_{n} \in B$ is called subadditive for a positive linear operator $T$ in $B$ if $F_{n+k} \leq F_{n}+T^{n} F_{k}$. We study the norm convergence of $n^{-1} F_{n}$ and its connection with weak convergence. Kingman's subadditive ergodic theorem states pointwise and $L_{1}$-norm convergence for $n^{-1} F_{n}$ under the condition $\sup \left\{n^{-1}\left\|F_{n}\right\|_{1}: n \geq 1\right\}<\infty$, when $T$ arises from a measure preserving transformation in a probability space.

Our study was mainly motivated by the following two questions. If, in Kingman's statement, the assumption $\sup \left\{n^{-1}\left\|F_{n}\right\|_{2}: n \geq 1\right\}<\infty$ is added, does convergence in the $L_{2}$-norm hold? If $T$ arises from a uniquely ergodic homeomorphism of a compact metric space and if the $F_{n}$ are continuous and non-negative, does uniform convergence of $n^{-1} F_{n}$ hold (as in the additive case)? More generally, one can ask under which conditions on $B$ and $T$ norm convergence of $n^{-1} F_{n}$ holds when it holds for averages.

Although it turns out that the answers to both preceding questions are negative, as is shown by precise examples in $\S 6$, several positive results can be obtained.

† Address for correspondence: Dr U. Krengel, Institüt für Mathematische Statistik, Lotzestrasse 13, 3400 Göttingen, West Germany. 
Under a special condition on $B$ which is satisfied by $L_{p}$ - spaces $(1 \leq p<\infty)$ but not by $C(X)$ or $L_{\infty}$, it is possible to extend the mean ergodic theorem to non-negative subadditive sequences when $T$ is a positive contraction: that is the existence of a weak cluster point for $n^{-1} F_{n}$ is equivalent to the norm convergence. This is done in $\S 4$. To achieve this we prove first in $\S 3$ that for power-bounded $T$ in a general Banach lattice the existence of a fixed weak cluster point $f$ for $\left(n^{-1} F_{n}\right)$ implies $\lim \left\|\left(n^{-1} F_{n}-f\right)^{+}\right\|=0,\left(F_{n}\right)$ being any subadditive sequence.

In $\S 5$, under the condition $\sup \left\{n^{-1}\left\|F_{n}\right\|\right\}<\infty$ we prove that $n^{-1} F_{n}$ converges weakly, when $T$ is an isometry of $L_{p}(1<p<\infty)$, and that $n^{-1} F_{n}$ converges in norm, when $B=L_{1}$ and $T$ is a positive contraction having a strictly positive fixed element.

Whenever possible our results are stated and proved directly for discrete $d$ parameter abelian semigroups of positive operators. A few results on a.e.-convergence are added, but in this direction several interesting questions remain unsolved.

\section{Preliminaries}

Let $d \geq 1$ be a fixed integer and let $\Im=\{0,1,2, \ldots\}$ be the additive semigroup of $d$-dimensional vectors with non-negative integer coordinates. For $a=\left(a_{i}\right)$, $b=\left(b_{i}\right) \in \Im$ we write $a \leq b$ if $a_{1} \leq b_{i}(i=1, \ldots, d)$ and $a<b$ if $a_{i}<b_{i}(i=1, \ldots, d)$. $\Im$ denotes the class of all non-empty intervals $[a, b)=\{u \in \Xi: a \leq u<b\}$. For $A \subset \subseteq|A|$ shall be the cardinality of $A$.

A linear operator $T$ in a Banach lattice $B$ with positive cone $B^{+}$is called positive if

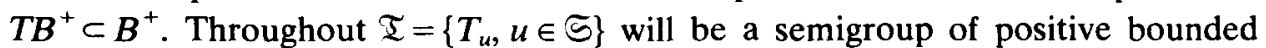
linear operators in $B$; in particular, we have $T_{u+v}=T_{u} \circ T_{v}(u, v \in \Im)$ and $T_{0}=$ identity, where $0=(0,0, \ldots, 0)$ is the neutral element in $\Im$. $T$ is called a contraction if $\|T\| \leq 1$.

A set function $F: \Im \ni I \rightarrow F_{I} \in B$ will be called a superadditive process (for $\mathfrak{T}$ ) if the following conditions are satisfied:

(2.1) $T_{u} F_{I}=F_{I+u}$ whenever $I \in \mathfrak{J}$ and $u \in \Im$.

(2.2) If $I_{1}, I_{2}$ are disjoint sets in $\Im$ and if $I_{1} \cup I_{2}$ is also in $\Im$ then $F_{I_{1} \cup I_{2}} \geq F_{I_{1}}+F_{I_{2}}$.

For short we write $F=\left\{F_{I}\right\}$. This definition does not contain the usual boundedness condition (see, for example, condition (1.1.3) in [13]) which is adapted to the case $B=L_{1}$. We shall impose boundedness conditions separately. In [2] the condition (2.2) was imposed for finitely many sets $I_{1}, \ldots, I_{k}$ since this was crucial for proofs of almost sure convergence, but here the slightly weaker formulation due to Smythe [15] is sufficient.

$F$ is called subadditive if $\left\{-F_{I}\right\}$ is superadditive, i.e. (2.2) holds with $\leq . F$ is called additive if it is both subadditive and superadditive.

For $n=\left(n_{1}, \ldots, n_{d}\right) \in \circlearrowleft$ let $\pi(n)=|[0, n)|=\prod_{\nu=1}^{d} n_{\nu}$ and $F_{n}=F_{[0, n)} . n \rightarrow \infty$ shall mean that $n_{\nu}$ tends to infinity for $\nu=1, \ldots, d$.

\section{Partial convergence for general subadditive processes}

An element $f \in B$ is called a fixed point for $\mathfrak{T}=\left\{T_{u}: u \in \Im\right\}$ or $\mathfrak{T}$-invariant if $T_{u} f=f$ holds for all $u \in \mathfrak{S}$. $\mathfrak{T}$ is called bounded if $\sup \left\{\left\|T_{u}\right\|: u \in \mathbb{S}\right\}<\infty$. 
THEOREM 3.1. If $F=\left\{F_{I}\right\}$ is a subadditive process for a bounded d-parameter semigroup $\mathfrak{T}$ of positive linear operators in a Banach lattice $B$ and the closed convex hull of $\left\{|I|^{-1} F_{I}: I \in \mathfrak{I}\right\}$ contains a fixed point f for $\mathfrak{T}$, then $\left\|\left(\pi(n)^{-1} F_{n}-f\right)^{+}\right\|$tends to zero as $n \rightarrow \infty$.

Proof. For any $\varepsilon>0$ there exist real numbers $\alpha_{1}, \alpha_{2}, \ldots, \alpha_{N} \geq 0$ with $\sum_{\nu=1}^{N} \alpha_{\nu}=1$ and intervals $I(\nu)=[a(\nu), b(\nu)) \in \mathfrak{S}$ such that

$$
\left\|\sum_{\nu=1}^{N} \alpha_{\nu}|I(\nu)|^{-1} F_{I(\nu)}-f\right\|<\varepsilon .
$$

Put

$$
\circlearrowleft(\nu)=\left\{t=\left(t_{1}, \ldots, t_{d}\right) \in \circlearrowleft: t_{i} \in\left(b_{i}(\nu)-a_{i}(\nu)\right) \cdot \mathbb{Z}^{+}\right\} .
$$

If $f_{n}, g_{n}$ are vectors in $B(n \in \subseteq)$ then $f_{n} \leq g_{n}+o(n)$ shall mean that there exist vectors $h_{n} \in B$ such that $f_{n} \leq g_{n}+h_{n}$ and $\pi(n)^{-1}\left\|h_{n}\right\| \rightarrow 0$ as $n \rightarrow \infty$. The subadditivity together with the boundedness of $\mathfrak{I}$ yield for each $k \in[0, b(\nu)-a(\nu))$ and for each $\nu=1, \ldots, N$ an estimate

$$
F_{n} \leq T_{k}\left(\sum_{\substack{u \in \Xi(\nu) \\ u<n-k-b(\nu)}} T_{u} F_{I(\nu)}\right)+o(n) .
$$

Adding over all such $k$ we obtain

It follows that

$$
|I(\nu)| F_{n} \leq \sum_{\substack{u \in \Xi \\ u<n-b(\nu)}} T_{u} F_{I(\nu)}+o(n)=\sum_{\substack{u \in \Xi \\ u<n}} T_{u} F_{I(\nu)}+o(n) .
$$

$$
F_{n}-\pi(n) f \leq \sum_{\substack{u \in \Xi \\ u<n}} T_{u}\left(\sum_{\nu=1}^{N} \alpha_{\nu}|I(\nu)|^{-1} F_{I(\nu)}-f\right)+o(n)
$$

or that

$$
\left(\pi(n)^{-1} F_{n}-f\right)^{+} \leq \pi(n)^{-1} \sum_{\substack{u \in \Xi_{1} \\ u<n}} T_{u}\left(\sum_{\nu=1}^{N} \alpha_{\nu}|I(\nu)|^{-1} F_{I(\nu)}-f\right)^{+}+\pi(n)^{-1} o(n) .
$$

Let $M=\sup \left\{\left\|T_{u}\right\|: u \in \widetilde{\Xi}_{1}\right\}$. As the sum on the right-hand side has $\pi(n)$ terms and each of them has norm at most $M \varepsilon$, we obtain that, for large $n$,

$$
\left\|\left(\pi(n)^{-1} F_{n}-f\right)^{+}\right\| \leq M \varepsilon+\varepsilon .
$$

As $\varepsilon$ was arbitrary the assertion follows.

Note that, by Mazur's theorem, the assumption on $f$ in theorem 3.1 is satisfied if $f$ is a weak cluster point of $\left\{|I|^{-1} F_{I}: I \in \Im\right\}$.

\section{Non-negative subadditive processes}

We now discuss the convergence of $\pi(n)^{-1} F_{n}$ for non-negative subadditive processes. We shall need some assumptions on the structure of the Banach lattice $B . B$ is called countably order complete (COC) if, for any non-empty countable subset $E \subset B$ which is majorized by an element of $B$, sup $E$ exists. We say that $B$ has an order continuous norm (OCN) if $B$ is COC and any decreasing sequence in $B^{+}$is 
norm-convergent. It has been pointed out by Ghoussoub \& Steele [11, prop. 1] that a COC Banach lattice $B$ has an order continuous norm if and only if, for every sequence $f_{n} \in B^{+}(n \in \mathbb{N})$ satisfying $f_{n+k} \leq f_{n}+f_{k}$ the sequence, $n^{-1} f_{n}$ norm-converges to $\inf n^{-1} f_{n}$.

THEOREM 4.1. Let $B$ be a Banach lattice with order continuous norm and let $\mathfrak{I}=\left\{T_{u}, u \in \subseteq\right\}$ be a bounded d-parameter semigroup of positive linear operators in $B$. If $F=\left\{F_{I}\right\}$ is subadditive and $F_{I} \geq 0$, then the weak convergence of $\pi(n)^{-1} F_{n}(n \rightarrow \infty)$ to a fixed point $f$ implies the strong convergence.

Proof. For any $h$ in the dual $B^{*}$ of $B, h\left(\pi(n)^{-1} F_{n}-f\right)$ tends to zero. By theorem 3.1 $h\left(\left(\pi(n)^{-1} F_{n}-f\right)^{+}\right)$also tends to zero. Thus $\left(\pi(n)^{-1} F_{n}-f\right)^{-}$must tend to zero weakly. We have $0 \leq\left(\pi(n)^{-1} F_{n}-f\right)^{-} \leq f$. By a lemma of Heinich [12] (see also [11]) a Banach lattice $B$ with order continuous norm has the following property: if $0 \leq x_{n} \leq x \in B$ and $x_{n}$ converges weakly to $x$, then $x_{n}$ converges strongly to $x$. We apply this lemma with $x_{n}=f-\left(\pi(n)^{-1} F_{n}-F\right)^{-}$and $x=f$.

In the additive case even the weak convergence to $f$ of a subsequence of $\pi(n)^{-1} F_{n}$ implies the strong convergence of $\pi(n)^{-1} F_{n}$; we shall prove below that this can be obtained here if $B$ satisfies a stronger property. The following example shows that the order-continuity of the norm is not sufficient to yield such a result:

Example 4.2. The space $B=C(X)$ with $X=\{0,1\}$ has an order continuous norm. The operator $T$ defined by $T f(0)=f(0), T f(1)=f(0)$ is a positive contraction. For any sequence $\varepsilon_{i}(i=0,1, \ldots)$ of zeros and ones the family $F_{n}(n \in \mathbb{N})$ defined by $F_{n}(0)=$ $n, F_{n}(1)=\sum_{i=0}^{n-1} \varepsilon_{i}$ determines a non-negative subadditive process for $\mathfrak{T}=\left\{T^{n}, n \geq 0\right\}$. It is easy to choose the $\varepsilon_{i} \mathrm{~s}$ in such a way that $n^{-1} F_{n}$ converges to the ( $T$-invariant) constant 1 along a subsequence but not along the entire sequence $n=1,2, \ldots$.

We shall now prove the desired stronger form of theorem 4.1 and several related interesting propositions for Banach lattices with uniformly monotone norm. Following the terminology of Birkhoff [5] the norm of a Banach lattice $B$ is called uniformly monotone if

for all $\varepsilon>0$ there exists $\delta>0$ such that for all $f, g \in B$ the properties $0 \leq f \leq g,\|f\|=1$, and $\|g\| \leq 1+\delta$ imply $\|g-f\|<\varepsilon$.

A Banach lattice having a uniformly monotone norm is called a UMB-lattice. The class of UMB-lattices contains the spaces $L_{p}(1 \leq p<\infty)$ and also the class of Banach lattices having uniformly convex norms. It is an exercise to show that UMB-lattices have an order continuous norm. In the sequel we shall use the following equivalent formulation of the definition of uniform monotonicity:

(4.1) for all $\varepsilon>0$ there exists $\delta>0$ such that for all $f, g \in B$ the properties $0 \leq f \leq g, 1-\delta \leq\|f\|$ and $\|g\| \leq 1+\delta$ imply $\|f-g\|<\varepsilon$.

We shall need

THEOREM 4.3. Let $\mathfrak{I}=\left\{T_{u}: u \in \mathbb{S}\right\}$ be a d-parameter semigroup of positive contractions acting on a UMB-lattice $B$. Let $\left\{F_{I}\right\}$ be a non-negative subadditive process for $\mathfrak{T}$. 
For any $w \in \mathbb{S}$ we have

$$
\lim _{n \rightarrow \infty} \pi(n)^{-1}\left\|F_{n+w}-F_{n}\right\|=0
$$

and

$$
\lim _{n \rightarrow \infty} \pi(n)^{-1}\left\|F_{n}-T_{w} F_{n}\right\|=0
$$

Proof. It is clear that it is enough to prove the assertions for $w=e(\nu)=$ $\left(e_{1}(\nu), e_{2}(\nu), \ldots\right)$ with $e_{i}(\nu)=\delta_{i \nu}(1 \leq i, \nu \leq d)$ where $\delta_{i \nu}$ is the Kronecker symbol. To prove the first assertion we observe that any non-negative subadditive process satisfies

$$
F_{n+e(\nu)} \leq F_{n}+\sum_{\substack{v<n+e(\nu) \\ v_{\nu}=n_{\nu}}} T_{v} F_{e}
$$

with $e=(1,1, \ldots, 1)$. Using the contraction property of the operators $T_{u}$ we can conclude

$$
\begin{aligned}
\pi(n)^{-1}\left\|\left(F_{n+e(\nu)}-F_{n}\right)^{+}\right\| & \leq \pi(n)^{-1}\left\|\sum_{\substack{\nu<n_{n+e(\nu)} \\
\nu_{\nu}=n_{\nu}}} T_{\nu} F_{e}\right\| \\
& \leq \pi(n)^{-1} \cdot\left(n_{1} \cdot n_{2} \cdots n_{\nu-1} \cdot n_{\nu+1} \cdots n_{d}\right)\left\|F_{e}\right\| \rightarrow 0 .
\end{aligned}
$$

Applying the identity $a=a \wedge b+(a-b)^{+}$we see that

$$
F_{n+e(\nu)}=\left(F_{n+e(\nu)} \wedge F_{n}\right)+\left(F_{n+e(\nu)}-F_{n}\right)^{+}
$$

and

$$
\left\|F_{n+e(\nu)}-F_{n}\right\| \leq\left\|F_{n}-\left(F_{n+e(\nu)} \wedge F_{n}\right)\right\|+\left\|\left(F_{n+e(\nu)}-F_{n}\right)^{+}\right\|,
$$

so that it is enough to prove $\pi(n)^{-1}\left\|F_{n}-\left(F_{n+e(\nu)} \wedge F_{n}\right)\right\| \rightarrow 0$.

As the operators $T_{u}$ are contractions, the function $I \rightarrow\left\|F_{I}\right\|$ is subadditive. Hence $\lim \pi(n)^{-1}\left\|F_{n}\right\|=\inf \left\{\pi(n)^{-1}\left\|F_{n}\right\|: n \in \mathbb{S}\right\}=\gamma \geq 0$. If $\gamma=0$ the theorem is obvious. If not we may and do assume that $\gamma=1$. Given $\varepsilon>0$ find $\delta>0$ from (4.1). From $1 \leq \pi(n+e(\nu)) \pi(n)^{-1} \rightarrow 1$ and (4.2) it follows that

$$
1 \leq \pi(n+e(\nu))^{-1}\left\|F_{n+e(\nu)}\right\| \leq \pi(n)^{-1}\left\|F_{n+e(\nu)} \wedge F_{n}\right\|+\pi(n)^{-1}\left\|\left(F_{n+e(\nu)}-F_{n}\right)^{+}\right\| .
$$

Therefore, taking $n$ large enough, we shall have

$$
1-\delta \leq \pi(n)^{-1}\left\|F_{n+e(v)} \wedge F_{n}\right\|
$$

and

$$
1 \leq \pi(n)^{-1}\left\|F_{n}\right\| \leq 1+\delta
$$

By the choice of $\delta$ this yields $\pi(n)^{-1}\left\|F_{n}-\left(F_{n+e(v)} \wedge F_{n}\right)\right\|<\varepsilon$ and the first assertion is proved.

The proof of the second assertion is similar. We have

$$
F_{n+e(\nu)} \leq T_{e(\nu)} F_{n}+\sum_{\substack{v<n+e(\nu) \\ v_{\nu}=0}} T_{v} F_{e}
$$

which implies $\pi(n)^{-1}\left\|\left(F_{n+e(\nu)}-T_{e(\nu)} F_{n}\right)^{+}\right\| \rightarrow 0$. By the same argument as above it is enough to prove that

$$
\pi(n)^{-1}\left\|F_{n+e(\nu)}-\left(F_{n+e(\nu)} \wedge T_{e(\nu)} F_{n}\right)\right\| \rightarrow 0 .
$$


By

$$
\begin{aligned}
1 & \leq \pi(n+e(\nu))^{-1}\left\|F_{n+e(\nu)}\right\| \\
& \leq \pi(n+e(\nu))^{-1}\left\|F_{n+e(\nu)} \wedge T_{e(\nu)} F_{n}\right\|+\pi(n+e(\nu))^{-1}\left\|\left(F_{n+e(\nu)}-T_{e(\nu)} F_{n}\right)^{+}\right\|
\end{aligned}
$$

we shall obtain

$$
1-\delta \leq\left\|F_{n+e(\nu)} \wedge T_{e(\nu)} F_{n}\right\|
$$

for large enough $n$, and then

$$
\pi(n+e(\nu))^{-1}\left\|\left(F_{n+e(\nu)} \wedge T_{e(\nu)} F_{n}\right)-F_{n+e(\nu)}\right\|<\varepsilon .
$$

Together with the first assertion this proves the second assertion.

Remarks. The theorem is not true if $B$ is not a UMB-lattice. This may be seen with the help of example 4.2. If $n^{-1} \sum_{i=0}^{n-1} \varepsilon_{i}$ does not converge to 1 then $n^{-1}\left\|F_{n}-T F_{n}\right\|$ fails to converge to zero. To show that the first assertion may also fail, consider the identity operator $T$ on $B=l_{\infty}(\mathbb{N})$ and $F_{n}=n 1_{[n, \infty[}$. Even if $B=L_{2}$ we also cannot replace the condition $\left\|T_{u}\right\| \leq 1$ in the theorem by the condition $\sup \left\{\left\|T_{u}\right\|: u \in \Xi\right\}<\infty$. To see this consider again example 4.2 , but replace the sup-norm by the $L_{2}$-norm. Then we have $\|T\|=2^{1 / 2}$ and $T^{n}=T$ for all $n$. We also cannot replace the condition $F_{n} \geq 0$ by the weaker condition sup $n^{-1}\left\|F_{n}\right\|<\infty$ even if $T$ is an isometry and $B=L_{2}$. This will be shown with the help of example 6.1.

We are now ready to prove the subadditive mean ergodic theorem for UMBlattices.

THEOREM 4.4. Let $\mathfrak{T}=\left\{T_{u}, u \in \Xi\right\}$ be a $d$-parameter semigroup of positive contractions in a UMB-lattice $B$ and $\left\{F_{I}: I \in \mathfrak{I}\right\}$ a non-negative subadditive process for $\mathfrak{T}$. For $f \in B$ the following assertions are equivalent:

(i) $f=\lim _{n \rightarrow \infty} \pi(n)^{-1} F_{n}$ in the norm topology.

(ii) $f$ is a weak cluster point of $\pi(n)^{-1} F_{n}$ when $n \rightarrow \infty$.

(iii) $f$ is fixed under $\mathfrak{T}$ and it belongs for all $v \in \mathbb{S}$ to the closed convex hull of $\left\{\pi(u)^{-1} F_{u}: u \geq v\right\}$.

Proof. The implication (i) $\Rightarrow$ (ii) is obvious.

(ii) $\Rightarrow$ (iii). By theorem 4.3, if $f$ is a weak cluster point of $\pi(n)^{-1} F_{n}$ where $n \rightarrow \infty$ then $f$ is fixed under $\mathfrak{T}$. By the Mazur theorem $f$ belongs for all $v \in \Xi$ to the closed convex hull of $\left\{\pi(u)^{-1} F_{u}: u \geq v\right\}$.

(iii) $\Rightarrow$ (i). Since $\lim _{n \rightarrow \infty} \pi(n)^{-1}\left\|F_{n}\right\|=\inf \left\{\pi(u)^{-1}\left\|F_{u}\right\|: u \in \Xi\right\}=\gamma \geq 0$ we have $\|f\| \leq \gamma$.

By theorem 3.1 we have $\left\|\left(\pi(n)^{-1} F_{n}-f\right)^{+}\right\| \rightarrow 0$. Since $\pi(n)^{-1} F_{n}=$ $\left(\left(\pi(n)^{-1} F_{n}\right) \wedge f\right)+\left(\pi(n)^{-1} F_{n}-f\right)^{+}$it is enough to prove that $\left\|f-\left(\pi(n)^{-1} F_{n}\right) \wedge f\right\| \rightarrow 0$. If $\gamma=0$ we have finished, if not we may again assume $\gamma=1$. Given $\varepsilon>0$ choose $\delta>0$ according to the UMB-condition (4.1). Since

$$
1 \leq \pi(n)^{-1}\left\|F_{n}\right\| \leq\left\|\pi(n)^{-1} F_{n} \wedge f\right\|+\left\|\left(\pi(n)^{-1} F_{n}-f\right)^{+}\right\|,
$$

we shall have

$$
1-\delta \leq\left\|\left(\pi(n)^{-1} F_{n}\right) \wedge f\right\| \leq\|f\| \leq 1
$$


for large enough $n \in \subseteq$. The UMB-condition now yields $\left.\| \pi(n)^{-1} F_{n}\right) \wedge f-f \|<\varepsilon$, which proves the theorem.

Our next aim is to derive some consequences of theorem 4.4. The example $B=L_{1}$ shows that $\pi(n)^{-1} F_{n}$ need not converge even in the additive case. We want to show that in our situation additive convergence implies subadditive convergence. To do this we need the following proposition which may also be of independent interest.

For $u=\left(u_{1}, \ldots, u_{d}\right), w=\left(w_{1}, \ldots, w_{d}\right) \in \subseteq$ we employ the notation

$$
u \circ w=\left(u_{1} w_{1}, u_{2} w_{2}, \ldots, u_{d} w_{d}\right) \text {. }
$$

Proposition 4.5. Let $B$ be a Banach-lattice with order continuous norm and let $\mathfrak{T}=\left\{T_{u}, u \in \subseteq\right\}$ be a bounded d-parameter semigroup of positive linear operators in $B$. Then for any $w>0$ and $f \in B^{+}$the following assertions are equivalent:

(i) $\pi(n)^{-1} \sum_{v<n} T_{v} f$ converges strongly when $n \rightarrow \infty$.

(ii) $\pi(n)^{-1} \sum_{v<n} T_{\text {wov }} f$ converges strongly when $n \rightarrow \infty$.

Proof. The implication (ii) $\Rightarrow$ (i) follows easily from

$$
\sum_{t<w} T_{t}\left(\sum_{v<n} T_{w \circ v} f\right)=\sum_{v<n \circ w} T_{v} f
$$

and holds in any Banach space without any positivity or norm-conditions.

(i) $\Rightarrow$ (ii). Since $f \geq 0, \sum_{v<n} T_{w \circ v} f \leq \sum_{v<w^{\circ} n} T_{v} f$. Hence with $h_{n}=\pi(n)^{-1} \sum_{v<n} T_{w \circ v} f$ and $g_{n}=\pi(w) \pi(n \circ w)^{-1} \sum_{v<w \circ n} T_{v} f$ we find that $0 \leq h_{n} \leq g_{n}$. By assumption, $g_{n}$ is converging strongly to some $g \in B^{+}$when $n \rightarrow \infty$. As $B$ has an order continuous norm, the set $\left\{h \in B^{+}: h \leq g\right\}$ is weakly compact by theorem 5.10 in [14]. It follows that the sequence $h_{n} \wedge g$ has a weak cluster point and $\left(h_{n}-g\right)^{+}$converges strongly to 0 . Hence $\left(h_{n} \wedge g\right)+\left(h_{n}-g\right)^{+}$has a weak cluster point when $n \rightarrow \infty$. From the general mean ergodic theorem [9] this is enough to imply (ii).

In general, the implication (i) $\Rightarrow$ (ii) is not true even for positive contractions in a Banach lattice $B$ without the condition that $B$ has an order continuous norm. To see this one can take $B=l_{\infty}(\mathbb{N})$ and $T f(k)=f(k+1)$ : it is not difficult to build a sequence $\left(\varepsilon_{i}\right)_{i \in \mathbb{N}}$ of zeros and ones such that the averages $n^{-1} \sum_{i=j}^{i+n-1} \varepsilon_{i}$ converge uniformly in $j$ but for which the implication (i) $\Rightarrow$ (ii) is false.

In this example the convergence holds only for certain elements $f \in B$. We do not know if for any positive contraction in an arbitrary Banach lattice the strong convergence of $n^{-1} \sum_{i=0}^{n-1} T^{i} f$ for all $f$ implies the strong convergence of $n^{-1} \sum_{i=0}^{n-1} T^{2 i} f$ for all $f$. At any rate such a conclusion is not true for arbitrary contractions in a Banach space. Consider the subspace $B$ of $L_{1}(\mathbb{Z})$ (with the counting measure) consisting of all elements $f$ with $\sum_{k \text { odd }} f(k)=-\sum_{k \text { even }} f(k)$. If $T$ is again the operator induced by the translation we have $n^{-1} \sum_{k=0}^{n-1} T^{k} f \rightarrow 0$ for all $f$, but there are elements $f \in B$ for which $n^{-1} \sum_{k=0}^{n-1} T^{2 k} f$ does not converge. 
THEOREM 4.6. Let $\mathfrak{T}=\left\{T_{u}: u \in \mathbb{S}\right\}$ be a d-parameter semigroup of positive contractions acting on a UMB-lattice $B$. Let $\left\{F_{I}\right\}$ be a non-negative subadditive process for $\mathfrak{T}$. If $\pi(n)^{-1} \sum_{v<n} T_{v} F_{e}$ converges strongly in $B$ when $n \rightarrow \infty$ then $\pi(n)^{-1} F_{n}$ also converges strongly. Furthermore, the following strong limits exist:

$$
\begin{aligned}
& \lim _{n \rightarrow \infty} \pi(n)^{-1} \sum_{v<n} T_{w \circ v} F_{w}=: \hat{F}_{w} \quad(w \in \Xi) . \\
& \lim _{w \rightarrow \infty} \pi(w)^{-1} \hat{F}_{w}=\lim _{n \rightarrow \infty} \pi(n)^{-1} F_{n} .
\end{aligned}
$$

Proof. From the definition of a non-negative subadditive process we obtain $0 \leq F_{n} \leq$ $\sum_{v<n} T_{v} F_{e}$. If $\pi(n)^{-1} \sum_{v<n} T_{v} F_{e}$ converges strongly the argument used in the proof of proposition 4.5 shows that $\pi(n)^{-1} F_{n}$ has a weak cluster point. Thus, by theorem 4.4, $\pi(n)^{-1} F_{n}$ converges strongly to some $f \in B$. By the preceding proposition $\pi(n)^{-1} \sum_{v<n} T_{w \nu v} F_{e}$ converges strongly for all $w \in \subseteq$. Since $0 \leq \sum_{v<n} T_{w \nu v} F_{w} \leq$ $\sum_{v<n} T_{\text {wov }}\left(\sum_{t<w} T_{t} F_{e}\right)$ we can again use the same argument to infer that the sequence $\pi(n)^{-1} \sum_{v<n} T_{w o v} F_{w}$ has a weak cluster point. Hence the limits $\hat{F}_{w}$ exist for all $w$. Let $\varepsilon>0$ be given. If $\|f\|=0$ we have, for large enough $w, \pi(w)^{-1}\left\|F_{w}\right\|<\varepsilon$ and therefore $\left\|\hat{F}_{w}\right\|<\varepsilon \pi(w)$. In this case both limits in (4.4) are zero. In the case $\|f\|>0$ we may assume $\|f\|=1$. Find $\delta>0$ from the UMB-condition (4.1). If $w$ is so large that $\pi(w)^{-1}\left\|F_{w}\right\|<1+\delta$ we have $\pi(w)^{-1}\left\|\hat{F}_{w}\right\|<1+\delta$. Now

$$
\begin{aligned}
f & =\lim _{n \rightarrow \infty} \pi(n)^{-1} F_{n}=\lim _{n \rightarrow \infty} \pi(n \circ w)^{-1} F_{n \circ w} \leq \lim _{n \rightarrow \infty} \pi(w)^{-1} \pi(n)^{-1} \sum_{v<n} T_{v \circ w} F_{w} \\
& =\pi(w)^{-1} \hat{F}_{w}
\end{aligned}
$$

implies that $\left\|f-\pi(w)^{-1} \hat{F}_{w}\right\|<\varepsilon$.

Remarks. (1) The examples given after theorem 4.3 show that in the preceding theorem neither the assumption UMB nor the assumption $\left\|T_{u}\right\| \leq 1$ can be removed.

(2) In theorem 4.3 the assumption of convergence $\pi(n)^{-1} \sum_{v<n} T_{v} F_{e}$ is satisfied if there is a $\mathfrak{T}$-invariant $p \in B^{+}$with $\left\|\left(F_{e}-k p\right)^{+}\right\| \rightarrow 0(k \rightarrow \infty)$. In this case for all $0 \leq g \leq k p$ the averages $\pi(n)^{-1} \sum_{v<n} T_{v} g$ converge strongly since they belong to the weakly compact order interval $[0, k p]$ and $F_{e}$ can be approximated by such $g s$. If $B$ is a function space it therefore suffices that there exists a strictly positive $\mathfrak{T}$-invariant $p \in B$.

\section{Subadditive processes in $L_{p}$}

We now drop the condition that the considered subadditive process $\left\{F_{1}\right\}$ be nonnegative, but we assume $B=L_{p}(1 \leq p<\infty)$. We shall prove theorems on normconvergence for $p=1$ and on weak convergence for $1<p<\infty$ and $d=1$. We first recall the following additive mean ergodic theorem for $p=1$.

THEOREM 5.1. Let $\mathfrak{T}=\left\{T_{u}, u \in \mathfrak{S}\right\}$ be a semigroup of (not necessarily positive) contractions in $L_{1}=L_{1}(\mu)$. Assume that there exists a strictly positive $h \in L_{1}$ such that 
$\left|T_{u} f\right| \leq h$ whenever $u \in \subseteq$ and $|f| \leq h$. Then for any $f \in L_{1}$ there exists $a \mathfrak{I}$-invariant $\bar{f} \in L_{1}$ such that $\pi(n)^{-1} \sum_{u<n} T_{u} f$ converges strongly to $\bar{f}$.

This follows from the general mean ergodic theorem [9], if one observes that $f$ can be approximated by $f_{k}=((-k h) \vee f) \wedge(k h)$, because all averages $\pi(n)^{-1} \sum_{u<n} T_{u} f_{k}$ lie in the weakly compact order interval $[-k h, k h]$. Alternatively, theorem 5.1 can be deduced from theorem 9 of Dunford \& Schwartz [8, p. 679]: passing to the new reference measure $h \cdot \mu$, i.e. using the isomorphism of $L_{1}(h \cdot \mu)$ and $L_{1}(\mu)$, we may assume that the $L_{1}$-norm and the $L_{\infty}$-norm of the operators $T_{u}$ is $\leq 1$, and therefore also the $L_{2}$-norm. One can then approximate $f$ with $L_{2}$-elements.

In the subadditive case it is natural to assume that the operators $T_{u}$ are positive. For non-negative $F_{I}$ we have then already obtained a positive result combining theorem 5.1 with theorem 4.6. For general $F_{I}$ the assumption on $h$ must be strengthened as may be seen by considering the operators $T_{u} f=0(u \neq 0)$. The following theorem has been proved by Kingman $(d=1)[13]$ and Smythe [15] in the case $T_{u} f=f \cdot \tau_{u}$, where the $\tau_{u}$ are measure preserving transformations in a probability space. Our proof is different.

THEOREM 5.2. Let $\mathfrak{T}=\left\{T_{u}, u \in \subseteq\right\}$ be a $d$-parameter semigroup of positive contractions in $L_{1}$. Assume that there exists a strictly positive $\mathfrak{T}$-invariant $h \in L_{1}$. If $\left\{F_{I}\right\}$ is subadditive and $\inf \left\{\pi(n)^{-1} \int F_{n} d \mu: n \in \Xi\right\}>-\infty$, then $\pi(n)^{-1} F_{n}$ converges in $L_{1}$-norm to a $\mathfrak{T}$-invariant limit $\vec{f}$.

Proof. It is convenient to prove the result for the superadditive process $\left\{-F_{I}\right\}$ instead; we therefore assume that $\left\{F_{1}\right\}$ is superadditive and $\sup \left\{\pi(n)^{-1} \int F_{n} d \mu: n \in \Xi\right\}<\infty$. Subtracting the additive process $\sum_{u \in I} T_{u} F_{e}$, for which theorem 5.1 applies, we may further assume $F_{I} \geq 0$. The existence of a strictly positive $T_{u}$-invariant $h$ implies that the operators $T_{u}$ are conservative and therefore Markovian, i.e. $\int T_{u} g d \mu=\int g d \mu$ holds for all $g \geq 0$. Therefore the $d$-parameter family of real numbers $\gamma_{n}=\int F_{n} d \mu(n \in \Xi)$ is superadditive, so that $\pi(n)^{-1} \gamma_{n}$ converges to

$$
\gamma=\sup \left\{\pi(n)^{-1} \gamma_{n}: n \in \Subset\right\} \quad \text { as } n \rightarrow \infty \text {. }
$$

For $k \in \mathbb{N}, \nu=1, \ldots, d$ and $n=\left(n_{1}, \ldots, n_{d}\right) \in \Im$ let $h_{\nu}(n, k)=\left[n_{\nu} / k\right]$ be the largest integer $\leq n_{\nu} / k$. Put $N(n, k)=k^{d} h_{1}(n, k) \cdots h_{d}(n, k)$. Then $\pi(n)^{-1} N(n, k) \rightarrow 1$ $(n \rightarrow \infty)$ for fixed $k$. If $I_{k, n}$ denotes the interval

$$
\left[0,\left(k \cdot h_{1}(n, k), k \cdot h_{2}(n, k), \ldots\right)[\right.
$$

then the superadditivity and non-negativity of $\left\{F_{I}\right\}$ yield

$$
F_{I_{k, n}} \geq \sum_{i_{1}=0}^{h_{1}(n, k)-1} \ldots \sum_{i_{d}=0}^{h_{d}(n, k)-1} T_{\left(k i_{1}, \ldots, k i_{d}\right)} F_{e \cdot k} .
$$

Denote the right-hand side by $\sum_{k, n}$. For any $\varepsilon>0$ we may find $k$ with $k^{-d} \gamma_{k e}>\gamma-\varepsilon$. 
By theorem 5.1, $N(n, k)^{-1} \sum_{k, n}$ converges in $L_{1}$-norm to some $g_{k} \in L_{1}$ invariant under $T_{k e}$.

As all $T_{u}$ are Markovian we have

$$
\int \sum_{k, n} d \mu=h_{1}(n, k) \cdots h_{d}(n, k) \int F_{k e} d \mu \geq(\gamma-\varepsilon) N(n, k)
$$

It follows that $\int g_{k} d \mu \geq(\gamma-\varepsilon)$. Using $\pi(n)^{-1} N(n, k) \rightarrow 1$ we obtain $\pi(n)^{-1} \sum_{k, n} \rightarrow g_{k}$. Therefore $F_{n} \geq \sum_{k, n}$ implies that

$$
\int\left(\pi(n)^{-1} F_{n}-g_{k}\right)^{-} d \mu \leq \int\left(\pi(n)^{-1} \sum_{k, n}-g_{k}\right)^{-} d \mu<\varepsilon
$$

holds for large enough $n$. On the other hand,

$$
\int\left(\pi(n)^{-1} F_{n}-g_{k}\right) d \mu \leq \gamma-\int k^{-d} F_{k e}<\gamma-(\gamma-\varepsilon)=\varepsilon .
$$

Together this yields $\left\|\pi(n)^{-1} F_{n}-g_{k}\right\|_{1}<3 \varepsilon$.

If $k, l$ are two integers with $k \gamma_{k e}>\gamma-\varepsilon$ and $l \gamma_{l e}>\gamma-\varepsilon$ we obtain that $\left\|g_{k}-g_{l}\right\|_{1}<$ $6 \varepsilon$. Thus the sequence $g_{k}$ is a Cauchy-sequence and converges in $L_{1}$ to some $\bar{f}$ with $\int \bar{f} d \mu=\gamma$. Clearly $\left\|\pi(n)^{-1} F_{n}-\bar{f}\right\|_{1} \rightarrow 0$.

It remains to prove the $\mathfrak{T}$-invariance of $\bar{f}$. Instead of working with $F_{k e}$ above we may do the same with $F_{u}, u=\left(u_{1}, u_{2}, \ldots, u_{d}\right) \in \Xi$, when $u_{\nu} \geq 1(\nu=1, \ldots, d)$. We then obtain functions $g_{u}$ invariant under $T_{u}$. Let $w \in \Xi$ be fixed. For large enough $u$ we have $\left\|g_{u}-\bar{f}\right\|_{1}<\varepsilon$ and $\left\|g_{u+w}-\bar{f}\right\|_{1}<\varepsilon$. Hence

$$
\begin{aligned}
\left\|T_{w} \bar{f}-\bar{f}\right\|_{1} & \leq\left\|T_{w} \bar{f}-T_{w} g_{u}\right\|_{1}+\left\|T_{u+w} g_{u}-T_{u+w} g_{u+w}\right\|_{1}+\left\|g_{u+w}-\bar{f}\right\|_{1} \\
& \leq \varepsilon+2 \varepsilon+\varepsilon .
\end{aligned}
$$

Thus $\bar{f}$ is $\mathfrak{T}$-invariant.

Remarks on almost-sure convergence. We say that $n \in \Im$ tends to infinity in a restricted way, and write $n(\rightarrow) \infty$, if there exist $0<\alpha<\beta<\infty$ such that $\alpha \leq n_{i} / n_{j} \leq \beta$ holds for all $n$ under consideration and all $1 \leq i, j \leq d$. The main result on a.e.convergence for $d$-parameter semigroups of contractions in $L_{1}$ in the additive case is:

THEOREM 5.3. Let $\mathfrak{T}=\left\{T_{u}, u \in \mathbb{S}\right\}$ be a d-parameter semigroup of contractions in $L_{1}(\mu)(\mu \sigma$-finite $)$ such that $\left\|T_{u} f\right\|_{\infty} \leq\|f\|_{\infty}$ holds for $u \in \mathbb{S}, f \in L_{\infty} \cap L_{1}$. Then the averages $\pi(n)^{-1} \sum_{u<n} T_{u}$ f converge a.e. for all $f \in L_{1}$ as $n(\rightarrow) \infty$.

This is a slight variant of a theorem of Brunel [6]. Brunel has studied only the case $n_{1}=n_{2}=\ldots=n_{d}$, but his maximal inequality is sufficient to yield theorem 5.3 without change of the argument. If the assumption on the $L_{\infty}$-norm is replaced by the assumption of existence of a strictly positive $h \in L_{1}$ with $|f| \leq h \Rightarrow\left|T_{u} f\right| \leq h$, the assertion of theorem 5.3 remains valid because we can pass to $L_{1}(h \cdot \mu)$. In the superadditive case we only have: 
Proposition 5.4. If $\mathfrak{T}=\left\{T_{u}, u \in \Im\right\}$ is a d-parameter semigroup of positive contractions in $L_{1}(\mu),\left\{F_{I}\right\}$ superadditive, sup $\left\{\pi(n)^{-1} \int F_{I} d \mu: n \in \Im\right\}<\infty$, and there exists a strictly positive $\mathfrak{T}$-invariant $h \in L_{1}$, then

$$
\liminf _{n(\rightarrow) \infty} \pi(n)^{-1} F_{n}=\bar{f} \quad \text { a.e. }
$$

where $\bar{f}$ is the $L_{1}$-limit.

Proof. It follows from $L_{1}$-convergence that $\lim$ inf $\leq \bar{f}$. To prove $\geq$ we may again assume $F_{I} \geq 0$. For all $k, N(k, n)^{-1} \sum_{k, n}$ tends to $g_{k}$ a.e. by theorem 5.3 as $n(\rightarrow) \infty$. Because $F_{n} \geq \sum_{k, n}$ and $\pi(n)^{-1} N(k, n) \rightarrow 1$ we obtain

$$
\liminf _{n(\rightarrow) \infty} \pi(n)^{-1} F_{n} \geq g_{k}
$$

for all $k$. Now $\bar{f}=\lim _{k} g_{k}$ yields the proposition.

In the 1-parameter case one can use theorem 2.1 of Akcoglu \& Sucheston [4] on the existence of exact dominants to show that the limit in (5.1) actually exists a.e. Extending Kingman's thecrem $(d=1)$ to $d \geq 1$ Akcoglu \& Krengel have recently shown [2] that $\pi(n)^{-1} F_{n}$ converges a.e. as $n(\rightarrow) \infty$ if $\left\{F_{I}\right\}$ is superadditive for operators $T_{u}$ given by measure preserving transformations $\tau_{u}$ and $T_{u} f=f \circ \tau_{u}$, provided - of course-that $\sup \left\{\pi(n)^{-1} \int F_{n} d \mu\right\}<\infty$. The case of Markovian operators $T_{u}$ with $\left\|T_{u} f\right\|_{\infty} \leq\|f\|_{\infty}\left(f \in L_{1} \cap L_{\infty}, u \in \Xi\right)$ seems to require completely new methods.

In the 1-parameter case the following observation may be an interesting complement to the results of Akcoglu \& Sucheston [4]. Let $T$ be a positive contraction in $L_{1}$ and let $\left\{F_{I}\right\}$ be subadditive and non-negative for $\mathfrak{T}=\left\{T^{k}, k \geq 0\right\}$. In this case

$$
\sum_{n=1}^{\infty}\left(F_{n+1}-F_{n}\right)^{+} \leq \sum_{n=1}^{\infty} T^{n} F_{e}<\infty
$$

on the dissipative part $D$, and this proves the a.e.-convergence of the sequence $F_{n}$ on $D$. Together with the results in [4] this implies that $F_{n} / \sum_{i=0}^{n-1} T^{i} p$ converges a.e. on $\left\{\sum_{i=0}^{\infty} T^{i} p>0\right\}$ for $p \in L_{1}^{+}$, i.e. the full statement of the Chacon-Ornstein theorem holds for such processes without the restrictions that $T$ be Markovian or $\left\{F_{I}\right\}$ additive on $D$.

Fava [10] has proved unrestricted convergence in theorem 5.3 under stronger integrability conditions, which are needed even in the case of measure preserving transformations. Smythe [15] has discussed the difficulties connected with unrestricted a.e.-convergence for subadditive processes.

Weak convergence in $L_{p}(1<p<\infty)$. We now consider positive contractions in $L_{p}$ and study questions of a.e.-convergence and weak convergence. We require the following lemma due to Akcoglu \& Sucheston [3, proposition 3.4 and corollary 3.5.]: 
LEMMA 5.5. Let $(\Omega, \mathscr{F}, \mu)$ be a $\sigma$-finite measure space, $1<p<\infty$, and let $T$ be $a$ positive contraction in $L_{p}$. Then $\Omega$ decomposes uniquely $(\bmod \mu)$ into two sets $E$ and $E^{c}$ having the following properties:

(i) $E$ is the support of a $T$-invariant function $h \in L_{p}^{+}$and the support of any such function is contained in $E$.

(ii) $E$ and $E^{c}$ are invariant sets, i.e. the subspaces $L_{p}(E)$ and $L_{p}\left(E^{c}\right)$ are both invariant under $T$.

THEOREM 5.6. Let $(\Omega, \mathscr{F}, \mu)$ be $\sigma$-finite, $1<p<\infty$, and let $\left\{F_{I}\right\}$ be a superadditive process for the positive contraction $T$ in $L_{p}$ satisfying $\sup \left\{n^{-1}\left\|F_{n}\right\|_{p}: n \in \mathbb{N}\right\}<\infty$. Then on the part $E$ defined in lemma $5.5, n^{-1} F_{n}$ converges a.e. If $T$ is even a positive isometry then $n^{-1} F_{n}$ converges weakly in $L_{p}$.

Proof. Because of Akcoglu's ergodic theorem [1] and the mean ergodic theorem we may subtract the additive process $\sum_{u \in I} T^{u} F_{1}$ and assume $F_{I} \geq 0$. It is clear from (ii) in the lemma that the processes $\left\{1_{E} F_{I}\right\}$ and $\left\{1_{E^{c}} F_{I}\right\}$ are then both non-negative and superadditive with $\sup \left\{n^{-1}\left\|1_{E} F_{n}\right\|_{p}\right\}<\infty$ and $\sup \left\{n^{-1}\left\|1_{E^{c}} F_{n}\right\|_{p}\right\}<\infty$. It therefore suffices to discuss the cases $\Omega=E$ and $\Omega=E^{c}$ separately. In the first case there exists a strictly positive $T$-invariant $h \in L_{p}$. Let $m$ denote the measure $h^{p} \mu$, i.e. $m(A)=$ $\int h^{p} d \mu$. It is a finite measure equivalent to $\mu$. Given $f \in L_{\infty}(m)$ define $S f=$ $h^{-1} T(f \cdot h)$. The operator $S$ is linear and positive and we have $S 1=1$. The function $h$ satisfies $\int h \cdot T^{*} h^{p-1} d \mu=\int T h \cdot h^{p-1} d \mu=\|h\|_{p}^{p}$. Since $h^{p-1}$ is the unique function $g$ in $L_{q}=L_{p / p-1}$ such that $\int h g d \mu=\|h\|_{p}^{p}$, we see that $T^{*} h^{p-1}=h^{p-1}$. Hence

$$
\int S f d m=\int h^{p-1} T(f h) d \mu=\int f h^{p} d \mu=\int f d m
$$

and $S$ can be extended as a Markovian operator in $L_{1}(m)$. Moreover, $S^{n} f=$ $h^{-1} T^{n}(f h)$, and $\left\{h^{-1} F_{I}\right\}$ is non-negative and superadditive for $\left\{S^{n}, n \in \mathbb{N}\right\}$. Because of

$$
\left\|h^{-1} F_{n}\right\|_{L_{1}(m)} \leq\|h\|_{L_{p}(\mu)}^{1 / p-1}\left\|F_{I}\right\|_{L_{p}(\mu)}
$$

the new process satisfies the boundedness condition $\sup \left\{n^{-1}\left\|h^{-1} F_{n}\right\|_{L_{1}(m)}\right\}<\infty$. Akcoglu \& Sucheston's extension of Kingman's theorem [4] now asserts the a.e.convergence of $n^{-1} h^{-1} F_{n}$ and the first statement is proved.

The weak convergence statement in the case $\Omega=E$ follows from the a.e.convergence and $\sup \left\{n^{-1}\left\|F_{n}\right\|_{p}\right\}<\infty$.

It remains to discuss the case $\Omega=E^{c}$. By assumption all the sequences $\left((n+j)^{-1} F_{n+j}\right)_{n \geq 1}(j \geq 0)$ are norm-bounded in $L_{p}$. Let $g_{0}$ be a weak cluster point of $\left(n^{-1} F_{n}\right)$. By the diagonal procedure there exists a strictly increasing sequence of integers $\left(n^{\prime}\right)$ such that, for any $j \geq 0$, the weak limit $g_{j}$ of $\left(n^{\prime}+j\right)^{-1} F_{n^{\prime}+j}$ in $L_{p}$ exists. We have $\left\|g_{j}\right\|_{p} \leq \sup \left\{\left\|n^{-1} F_{n}\right\|_{p}\right\}$ and $g_{j} \geq 0$. From $F_{k+1} \geq F_{k}+T^{k} F_{1}$ and $\lim _{k} k^{-1}\left\|T^{k} F_{1}\right\|_{p}=0$ we obtain $g_{j+1} \geq g_{j}(j \geq 0)$. Therefore the strong limit $g$ of $\left(g_{j}\right)$ exists in $L_{p}$. Since the strong continuity of $T$ implies the weak continuity, we obtain 
$g_{j+1} \geq T g_{j}$ from $F_{k+1} \geq F_{1}+T F_{k}$ and $\lim k^{-1}\left\|F_{1}\right\|=0$. Taking the limit we obtain $g \geq T g$. As we have assumed that $T$ is an isometry in the second assertion, $g=T g$. Since the support of any $T$-invariant $g \geq 0$ was contained in $E$ and now $\Omega=E^{c}$ we must have $g=0$, and also $g_{0}=0$ since $g_{0} \leq g$. Therefore the sequence $n^{-1} F_{n}$ has only 0 as a weak cluster point in $L_{p}$. This proves the weak convergence to 0 .

Remarks. Using the operator $T f=0\left(f \in L_{p}\right)$ it is easy to see that the second assertion does not hold for all contractions. The norm-convergence may fail in both cases considered above as will be shown in the next section. The question of a.e.convergence in the case $\Omega=E^{c}$ remains unsolved. In the additive case Akcoglu [1] has proved a strong maximal inequality: if $T$ is a positive contraction in $L_{2}$ there is a constant $c_{p}<\infty$ such that $\left\|\sup n^{-1} F_{n}\right\|_{p} \leq c_{p}\left\|F_{1}\right\|_{p}$. In the superadditive non-negative case one might hope for $\left\|\sup n^{-1} F_{n}\right\|_{p} \leq c_{p} \delta_{p}$ with $\delta_{p}=\sup \left\|n^{-1} F_{n}\right\|_{p}$, but this fails even in the case where the operator is given by a measure preserving transformation. It is even possible that $\delta_{2}<\infty$ and $\mu\left(\left\{\sup n^{-1} F_{n}>1\right\}\right)=\infty$.

\section{Main counterexamples}

In this section we collect the main examples promised in the introduction and in the previous sections.

Example 6.1. We first show that there exists a measure preserving transformation $\tau$ in a probability space $(\Omega, \mathscr{F}, \mu)$ and a non-negative superadditive process $\left\{F_{I}\right\}$ for $\mathfrak{T}=\left\{\tau^{n}, n \geq 0\right\}$ such that $\sup \left\{n^{-1} \int F_{n} d \mu: n \geq 1\right\}$ and $\sup \left\{n^{-1}\left\|F_{n}\right\|_{2}: n \geq 1\right\}$ are finite but $n^{-1} F_{n}$ does not converge in $L_{2}$-norm.

We shall inductively define a sequence of integers $n_{1}<n_{2}<\cdots$. The space $\Omega$ shall be the union of the spaces $\Omega_{k}=\left\{(k, i): 1 \leq i \leq 2^{k} n_{k}\right\}$. Put $\tau(k, i)=(k, i-1)$ for $i>1$ and $\tau(k, 1)=\left(k, 2^{k} n_{k}\right)$, i.e. $\tau$ permutes $\Omega_{k}$ cyclically. The measure will be given by $\mu(\{(k, i)\})=2^{-2 k} n_{k}^{-1}$. Let $f_{k}$ be the function with $f_{k}((k, 1))=2^{k} n_{k}, f_{k}((j, i))=0$ otherwise. Once we know $n_{k}$ expressions like $n^{-1} \sum_{i=0}^{n-1} f_{k} \circ \tau^{i}$ make sense even though the definition of $\Omega$ is not yet complete. This average will be zero on the undefined part of $\Omega$. Start with $n_{1}=1$. When $n_{k}$ is defined find $n_{k+1}>n_{k}$ so that

for all $n \geq n_{k+1}, j \leq k$. Put

$$
\left\|n^{-1} \sum_{i=0}^{n-1} f_{i} \tau^{i}-1_{\Omega_{i}}\right\|_{2}<2^{-k}
$$

$$
F_{n}=\sum_{i=0}^{n-1}\left(f_{1}+\cdots+f_{k}\right) \circ \tau^{i}
$$

when $n_{k} \leq n<n_{k+1}$. Using $F_{I} \circ-^{i}=F_{I+k}$ this defines all $F_{I}$ and the process $\left\{F_{I}\right\}$ is superadditive. $\int f_{k} d \mu=2^{-k}$ implies the finiteness of $\sup \left\{n^{-1} \int F_{n} d \mu\right\}$. To show that the sequence $n^{-1}\left\|F_{n}\right\|_{2}$ is bounded first note that, for $n \geq n_{k}$ and $j \leq k-1$, we have

$$
\left\|n^{-1} \sum_{i=0}^{n-1} f_{j} \circ \tau^{i}\right\|_{2} \leq\left\|1_{\Omega_{j}}\right\|_{2}+2^{-k} \leq 2^{-j / 2}+2^{-k}
$$


which implies

$$
\left\|n^{-1} \sum_{j=1}^{k-1} \sum_{i=0}^{n-1} f_{j} \circ \tau^{i}\right\|_{2} \leq \sum_{j=1}^{\infty} 2^{-j / 2}+1<\infty .
$$

To estimate the contribution of $f_{k}$ we use that, for $n_{k} \leq n \leq 2^{k} n_{k}$,

$$
\begin{aligned}
n^{-1}\left\|_{i=0}^{n-1} f_{k} \circ \tau^{i}\right\|_{2} & =n^{-1}\left(n \cdot 2^{2 k} n_{k}^{2} 2^{-2 k} n_{k}^{-1}\right)^{1 / 2} \\
& =\left(n^{-1} n_{k}\right)^{\frac{1}{2}} \leq 1 .
\end{aligned}
$$

For general $n$ with $n_{k} \leq n<n_{k+1}$ we can put the sum $\sum_{i=0}^{n-1} f_{k} \circ \tau^{i}$ together from pieces with at least $n_{k}$ and at most $2^{k} n_{k}$ terms. Therefore

$$
\left\|n^{-1} \sum_{i=0}^{n-1} f_{k} \circ \tau^{i}\right\|_{2} \leq 1 .
$$

Together with the estimate (6.1) this proves the boundedness of the sequence $n^{-1}\left\|F_{n}\right\|_{2}$. It follows from

$$
\begin{aligned}
n_{k}^{-1}\left\|F_{n_{k}}-F_{n_{k}-1}\right\|_{2} & =n_{k}^{-1}\left\|\left(f_{1}+\cdots+f_{k-1}\right) \circ \tau^{n_{k}-1}+\sum_{i=0}^{n_{k}-1} f_{k} \circ \tau^{\|}\right\|_{2} \\
& \geq n_{k}^{-1}\left\|\sum_{i=0}^{n_{k}-1} f_{k} \circ \tau^{i}\right\|_{2}=1
\end{aligned}
$$

that the sequence $n^{-1} F_{n}$ does not converge in $L_{2}$.

The process $\left\{-F_{I}\right\}$ is subadditive. Therefore this example also shows that the first assertion in theorem 4.3 does not remain true if the non-negativity condition for the subadditive process is replaced by the boundedness condition

$$
\sup \left\{\pi(n)^{-1}\left\|F_{n}\right\|: n \in \Xi\right\}<\infty .
$$

It is easy to give also an example to this extent for the second assertion in theorem 4.3. Just define $F_{n}$ as above for $n \neq n_{k}$ and for $n=n_{k}$ put

$$
F_{n}=\sum_{i=0}^{n-1}\left(f_{1}+\cdots+f_{k-1}\right) \circ \tau^{i}+\sum_{i=0}^{[n / 2]-1} f_{k} \circ \tau^{2 i} .
$$

In the above example $\tau$ is not ergodic. It is not difficult to give a similar example with an ergodic $\tau$ in $[0,1]$ with Lebesgue-measure. The necessary sets $\Omega_{k}$ are then replaced by $2^{k} n_{k}$ disjoint images of suitable sets $E_{k}$ obtained with the help of Rohlin's lemma.

Example 6.2. In the terminology of lemma 5.5, example 6.1 shows that for non-negative superadditive $F_{n}$ the sequence $n^{-1} F_{n}$ need not converge in $L_{2}$ in the case $\Omega=E$. To find an example with $\Omega=E^{c}$ take $\Omega=\mathbb{R}^{1}, \mu=$ Lebesgue-measure, and let $\tau$ be given by $\tau \omega=\omega+1$. Start with some $f_{1} \geq 0$ with $\left\|f_{1}\right\|_{1} \leq 2^{-1}$ and $\left\|f_{1}\right\|_{2}=1$. There exists an $n_{1} \in \mathbb{N}$ such that $n \geq n_{1}$ implies $\left\|n^{-1} \sum_{k=0}^{n-1} f_{1} \circ \tau^{k}\right\|_{2}<2^{-1}$. Find $f_{2} \geq 0$ with $\left\|f_{2}\right\|_{1} \leq 2^{-2},\left\|\left(n_{1}+1\right)^{-1} \sum_{k=0}^{n_{1}} f_{2} \circ \tau^{k}\right\|_{2}=1$ and $\left\|n^{-1} \sum_{k=0}^{n-1} f_{2} \circ \tau^{k}\right\|_{2} \leq 1$ for $n \geq n_{1}+1$. When $n_{1}, \ldots, n_{k}, f_{1}, \ldots, f_{k+1}$ have been constructed, find $n_{k+1}$ such that $n \geq n_{k+1}$ 
implies $\left\|n^{-1} \sum_{i=0}^{n-1}\left(f_{1}+\cdots+f_{k+1}\right) \circ \tau^{i}\right\|_{2}<2^{-(k+1)}$. Then find $f_{k+2} \geq 0$ with

$$
\left\|f_{k+1}\right\|_{1}<2^{-(k+1)},\left\|\left(n_{k+1}+1\right) \sum_{i=0}^{n_{k+1}} f_{k+2} \circ \tau^{i}\right\|_{2}=1
$$

and

$$
\left\|n^{-1} \sum_{i=0}^{n-1} f_{k+2} \circ \tau^{i}\right\|_{2} \leq 1\left(n \geq n_{k+1}+1\right)
$$

Put $F_{n}=\sum_{i=0}^{n-1}\left(f_{1}+\cdots+f_{k+1}\right) \circ \tau^{i}$ for $n_{k}<n \leq n_{k+1}$. It is easy to check the desired properties of the process.

Example 6.3. If $\tau$ is a uniquely ergodic transformation (see [7]) in a compact metric space $\Omega$ and $f: \Omega \rightarrow \mathbb{B}$ continuous, then $n^{-1} \sum_{i=0}^{n-1} f \circ \tau^{i}$ converges uniformly. The following example shows that this classical result does not extend to non-negative subadditive processes, i.e. we construct a nön-negative subadditive process $\left\{F_{I}\right\}$, for which the $F_{I}$ are continuous, but $n^{-1} F_{n}$ fails to converge uniformly.

Let $\tau$ be any uniquely ergodic transformation in a compact metric space $\Omega$ such that the unique invariant probability measure $\mu$ is non-atomic. We construct a sequence of continuous non-negative functions $f_{0}, f_{1}, \ldots$ and a sequence $0=n_{0}<n_{1}<\ldots$ of integers. Let $\sum_{n, k}=\sum_{i=0}^{n-1}\left(f_{0}-f_{1}-f_{2}-\ldots-f_{k}\right) \circ \tau^{i}$. We shall put $F_{n}=\sum_{n, k}$ for $n_{k} \leq n<n_{k+1}$.

We start with $f_{0} \equiv 1, n_{1}=1$ and any continuous $f_{1}$ with $0 \leq f_{1} \leq 2^{-1}$ and $\int f_{1} d \mu<$ $2^{-2}$. After the $k$ th step we shall know $n_{k}$ and $f_{k}$ and we shall have $0 \leq f_{k} \leq 2^{-1}(1 \leq k)$ and $\int f_{k} d \mu<2^{-(k+1)}$. We find $n_{k+1}>n_{k}$ so large that $n \geq n_{k+1}$ implies

$$
\left|n^{-1} \sum_{n, k}-\int\left(f_{0}-f_{1}-\cdots-f_{k}\right) d \mu\right|<2^{-(k+2)}
$$

uniformly on $\Omega$. In particular, for $n \geq n_{k+1}$, we then have that $n^{-1} \sum_{n, k}>2^{-1}$ on $\Omega$. With the help of Rohlin's lemma we now find a measurable set $E_{k}$ with $\mu\left(E_{k}\right)>0$ such that the sets $\tau^{-i} E_{k}\left(i=0,1,2, \ldots, n_{k+1} 2^{(k+2)}\right)$ are disjoint.

Let $U_{k+1}=\underbrace{n_{k+1}-1}_{i=0} \tau^{-i} E_{k} . f_{k+1}$ shall be any continuous function with $0 \leq f_{k+1} \leq \frac{1}{2}$ which approximates $2^{-1} 1_{U_{k+1}}$ in $L_{1}$-norm so closely that $\int f_{k+1} d \mu<2^{-(k+2)}$ and $n_{k+1}^{-1} \sum_{i=0}^{n_{k+1}-1} f_{k+1} \circ \tau^{i}$ is $\geq 4^{-1}$ on a set of positive measure. This implies

$$
\left|F_{n_{k+1}-1}-F_{n_{k+1}}\right| \geq 4^{-1} n_{k+1}-(k+1) \text {. }
$$

We may assume $4^{-1} n_{k+1}-(k+1) \geq 8^{-1} n_{k+1}$. Thus $n^{-1} F_{n}$ cannot converge uniformly. 
This research was conducted during a visit of the first named author to the University of Göttingen.

\section{REFERENCES}

[1] M. A. Akcoglu. A pointwise ergodic theorem in $L_{p}$-spaces. Can. J. Math. 27 (1975), 1075-1082.

[2] M. A. Akcoglu \& U. Krengel. Ergodic theorems for super-additive processes. J. Reine u. Ang. Math. (in the press).

[3] M. A. Akcoglu \& L. Sucheston. On convergence of iterates of positive contractions in $L_{p}$-spaces. $J$. Approx. Theory 13 (1975), 348-362.

[4] M. A. Akcoglu \& L. Sucheston. A ratio ergodic theorem for super-additive processes. Z. Wahrscheinlichkeitstheorie verw. Geb. 44 (1978), 269-278.

[5] G. Birkhoff. Lattice Theory. Amer. Math. Soc. Coll. Publ. XXV: Providence, R.I., 1940.

[6] A. Brunel. Théorème ergodique ponctuel pour un semigroupe commutatif finiment engendré de contractions de $L_{1}$. Ann. Inst. Henri Poincaré 9 (1973), 327-343.

[7] M. Denker, C. Grillenberger and K. Sigmund. Ergodic Theory on Compact Spaces. Springer Lecture Notes in Math. no. 527. Springer: Berlin, 1976.

[8] N. Dunford \& J. T. Schwartz. Linear Operators Vol. 1. Interscience Publ.: New York, 1958.

[9] W. F. Eberlein. Abstract ergodic theorems and weak almost periodic functions. Trans. Amer. Math. Soc. 67 (1949), 217-240.

[10] N. A. Fava. Weak type inequalities for product operators. Studia Math. 42 (1972), 271-288.

[11] N. Ghoussoub \& J. M. Steele. Vector valued subadditive processes and applications in probability. Ann. Prob. 8 (1980), 83-95.

[12] H. Heinich. Convergence of positive submartingales in a Banach lattice. C.R. Acad. Sci. (Paris) Sec. A 286 (1978), 279-280.

[13] J. F. C. Kingman. Subadditive ergodic theory. Ann. Prob. 1 (1973), 883-905.

[14] H. H. Schaefer. Banach Lattices and Positive Operators. Springer: New York, 1975.

[15] R. T. Smythe. Multiparameter subadditive processes. Ann. Prob. 4 (1976), 772-782. 